\title{
Amperometric detection of dopamine and ascorbic acid with zeolite-graphite composite microelectrodes
}

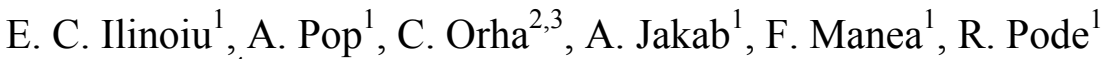 \\ \& P. A. Serra \\ ${ }^{1}$ Faculty of Industrial Chemistry and Environmental Engineering, \\ "Politehnica" University of Timisoara, Timisoara, Romania \\ ${ }^{2}$ The Institute of Research for Condensed Matter, Timisoara, Romania \\ ${ }^{3}$ National Institute of Research and Development in Microtechnologies, \\ Bucharest, Romania \\ ${ }^{4}$ Department of Neuroscience, Medical School, \\ University of Sassari, Italy
}

\begin{abstract}
Natural and synthetic zeolites were used to modify synthetic graphite based composite microelectrodes envisaging their use for simultaneous/selective detection of ascorbic acid (AA) and dopamine (DA) in aqueous solutions. Both natural and synthetic zeolite-modified synthetic graphite composite microelectrodes (ZN-GEC and SZ-GEC) were obtained in epoxy matrices, and the optimum composition was selected based on the electrochemical behavior. The composite electrodes were characterized electrochemically by cyclic voltammetry $(\mathrm{CV})$ using the classical ferrycianide/ferrocyanide system method, in comparison with bare graphite composite microelectrode (GEC). The electrochemical oxidation of AA occurred at all three microelectrodes and the slowest kinetics was noticed for SZ-GEC, due to the synthetic zeolite presence with certain peculiarities in relation to the selectivity and the catalytic effect, while ZN-GEC and GEC microelectrodes exhibited very good sensitivity for AA detection in aqueous solution. Taking into consideration the fact that DA and AA coexist in real extracellular brain fluid and the fact that SZ-GEC microelectrode did not exhibited a good sensitivity towards AA detection, but a very good sensitivity for DA was obtained, makes this composite material a good
\end{abstract}


candidate with real potential for selective determination of DA without a significant interference of AA.

Keywords: microelectrodes, dopamine, ascorbic acid, voltammetric detection, amperometric detection.

\section{Introduction}

Developing smaller geometries for working electrodes led implicitly to some important advantages, e.g. decreased ohmic drop of potential, fast establishment of a steady state signal, useful current increase, increased signal-to-noise ratio. These characteristics make small electrodes to be applicable in many areas of electro analytical chemistry and especially for in-vivo voltammetry and amperometry [1]. Gold, platinum and carbon have been used extensively [2] as electrode materials for sensing, although using a carbon material as conducting phase within various composite materials [3] produce a lot of benefits [4-6].

The main drawback with carbon composites is the slow electron transfer kinetics due to the presence of the non-conducting binder, which can be solved by the incorporation of specific catalytic materials in the composite matrix. The mixture of zeolite and graphite has been proposed since 1980s in various forms [7], leading to a class of electrodes named zeolite modified electrodes (ZMEs) [8].

Using charge selectivity property of the zeolite, the permselective detection of positively charged neurotransmitters in the presence of ascorbic acid was exploited using ZME [9]. Also, detection of ascorbic acid (AA) in the presence or not of dopamine (DA) has been researched using epoxy-carbon composite electrodes [10] and good results were obtained also by the use of different zeolite materials in composite matrices [11].

The present paper focuses on developing a new design and construction of graphite-epoxy and graphite-zeolite-epoxy composite microelectrode materials aiming in vitro electrochemical detection of ascorbic acid and dopamine neurotransmitters. The electrochemical behavior and the response of the designed materials to the presence of ascorbic acid and dopamine without any protective membranes were studied by cyclic voltammetry technique.

\section{Experimental}

\subsection{Chemicals and reagents}

Potassium ferrocyanide and Araldite $\mathrm{M}$ three-component epoxy resin (resin:hardener:accelerator) used in this study were purchased from Sigma. LAscorbic Acid (Sigma, Aldrich) solutions were freshly prepared prior to each experiment and dissolved in $0.01 \mathrm{M} \mathrm{HCl}$ solution, as well as protected against light exposure during experiments. Synthetic graphite powder $(<20 \mu \mathrm{m})$ was purchased from Sigma.

Sodium phosphate saline buffer solutions (PBS) supporting electrolyte was prepared from di-hydrogenphospate, sodium chloride and sodium hydroxide of 
analytical regent grade (Sigma, Aldrich) and used as received. PBS solution was kept in the refrigerator.

Natural zeolite with high clinoptilolite content and a mass composition consisted of: $62.20 \% \mathrm{SiO}_{2}, 11.65 \% \mathrm{Al}_{2} \mathrm{O}_{3}, 1.30 \% \mathrm{Fe}_{2} \mathrm{O}_{3}, 3.74 \% \mathrm{CaO}, 0.67 \%$ $\mathrm{MgO}, 3.30 \% \quad \mathrm{~K}_{2} \mathrm{O}, 0.72 \% \mathrm{Na}_{2} \mathrm{O}, 0.28 \% \mathrm{TiO}_{2}$, was supplied by Cemacon Company, Romania. Natural zeolite was used as Si source for synthetic zeolite preparation using sodium hydroxide solution and sodium aluminate as aluminium source in according with a previous-reported method [12]. The surface morphology of synthetic zeolite is similar to natural zeolite, revealing a more uniform structure with smooth edged lamellar crystals. The particles size of the natural zeolite ranged from 4 to $11 \mu \mathrm{m}$ and the particles size of the synthetic zeolite A was in the range from 20 to $300 \mathrm{~nm}$. Si/Al ratio was 4-5 for the natural zeolite and 1.0-1.14 for the synthetic zeolite.

\subsection{Apparatus and experimental measurements}

All voltammetric experiments were carried out at room temperature $\left(25^{\circ} \mathrm{C}\right)$ using an eDAQ QuadStat four-channel Potentiostat (Australia) controlled with an eChem software for cyclic voltammetry in a three-electrode electrochemical cell, carbon composite working electrodes, $\mathrm{Ag} / \mathrm{AgCl}$ reference electrode and $\mathrm{Pt}$ auxiliary electrode. A magnetic stirrer provided the convective transport during the voltammetric experiments.

The surface active area was investigated by cyclic voltammetry in a $20 \mathrm{~mL}$ volume electrochemical cell, in which a concentration of $2 \mathrm{mM} \mathrm{K}_{3} \mathrm{Fe}(\mathrm{CN})_{6} /$ $\mathrm{K}_{4} \mathrm{Fe}(\mathrm{CN})_{6}$ was added in a $1 \mathrm{M} \mathrm{KNO}_{3}$ supporting electrolyte. Cyclic voltammetry (CV) experiments were carried out by varying the scan rates from $25 \mathrm{mV} \mathrm{s}^{-1}$ to $454 \mathrm{mV} \mathrm{s}^{-1}$. The detection experiments were carried out using standard addition method in $50 \mathrm{mM}$ PBS supporting electrolyte at the scan rate of $250 \mathrm{mV} \mathrm{s}^{-1}$.

\subsection{Sensor design and composite material construction}

The design of the sensor is presented in Figure 1. It was made using a Classical Multi Core $\mathrm{Cu}$ wire $(5 \mathrm{~cm}$ in length, i.d. $=150 \mu \mathrm{m})$. Its plastic insulator was removed at one edge and $2.2 \mathrm{~cm}$ of $\mathrm{Cu}$ wire was exposed and inserted in a Fused Silica Tube (i.d. $=180 \mu \mathrm{m})$. At one end, $1 \mathrm{~mm}$ of Silica tube was left free in order to allow the filling with the graphite-epoxy composite, while the $\mathrm{Cu}$ wire guaranteed the electrical contact with the composite matrix. The geometrical area of the sensor surface with disc geometry of $2.510^{-4} \mathrm{~cm}^{2}$ and the final length of the sensor used for all electrochemical detections was approximately $5 \mathrm{~cm}$.

Carbon-epoxy based composites were constructed according to the following procedure. All sensors were prepared by manually mixing the graphite particles and zeolites with the three-component epoxy resin for about 20-30 min. Before curring, the material was filled inside the Si capillary (by dipping the sensor body into the composite) and the electrical contact of the composite with the copper wire was checked under microscope, as well as the exterior walls of the Si cappillary in order to be clean, to not interfere later in the voltammetric experiments. 


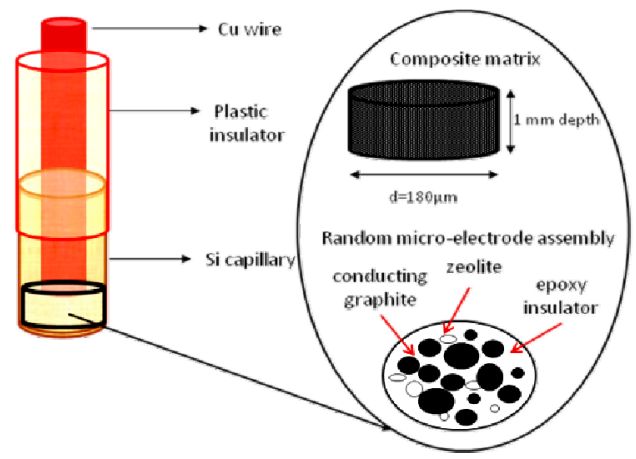

Figure 1: $\quad$ Sensor design.

A bare graphite-epoxy composite (GEC) sensor was firstly elaborated and considered as blank sensor, after finding the proper conditions for the threecomponent epoxy resin polymerization (curring at $105^{\circ} \mathrm{C}$ for $30 \mathrm{~min}$ ). In order to estimate the best three-component epoxy ratio (resin:hardener:accelerator) and the best mixing method in terms of the electrochemical behaviour, e.g. reversibility (peak-to-peak separation, $\Delta E$ ) and sensitivity (peak current, $\Delta I$ ), cyclic voltammetry was performed at a scan rate of $100 \mathrm{mV} \mathrm{s}^{-1}$ within potential range of -0.4 and $+0.8 \mathrm{~V}(v s$. $\mathrm{Ag} / \mathrm{AgCl})$ for $2 \mathrm{mM}$ potassium ferri/ferrocyanide system. Only two mixing methods and two resin ratios (100:95:5 and 100:10:5 resin:hardener:accelerator) were evaluated as concrete criteria of appreciation. The best graphite-epoxy weight ratio was found to be 55\% wt. graphite and $45 \%$ wt. epoxy with 100:95:5 resin:hardener:accelerator weight ratio, using the same voltammetric procedure.

The third material in the composite matrix was incorporated by mechanical dispersion of a constant $20 \%$, wt. natural or syntetic zeolite, while the graphite and epoxy concentrations were varried, resulting two new ZMEs: graphite 45\%natural zeolite 20\%-epoxy 35\%, wt. (NZ-GEC) and graphite 45\%-synthetic zeolite 20\%-epoxy 35\%, wt. (SZ-GEC).

Prior to be used, the hardened composite material surface was polished using a mechanical drill and checked again under microscope for a perfect disc shape of the microelectrode.

\section{Results and discussions}

\subsection{Electrochemical surface characterisation}

Cyclic voltammetry was used to determine the electroactive areas of all three GEC, NZ-GEC and SZ-GEC sensors by classical potassium ferri/ferrocyanide system in $1 \mathrm{M} \mathrm{KNO}_{3}$ supporting electrolyte recorded at different scan rates. Based on Randles-Sevcik equation:

$$
I_{p}=2.69 \times 10^{5} A D^{1 / 2} n^{3 / 2} v^{1 / 2} C
$$


where: $A$ represents the area of the electrode $\left(\mathrm{cm}^{2}\right), n$ is the number of electrons participating in the reaction and is equal to $1, D$ is the diffusion coefficient of the molecule in solution, $C$ is the concentration of the probe molecule in the solution and in this experimental set-up is $2 \mathrm{mM}$, and $v$ is the scan rate $\left(\mathrm{V} \mathrm{s}^{-1}\right)$, the apparent diffusion coefficient of $\mathrm{K}_{3} \mathrm{Fe}(\mathrm{CN})_{6}$ was determined to be $1.1110^{-4} \mathrm{~cm}^{2}$ $\mathrm{s}^{-1}$ for the GEC sensor, $3.5210^{-5} \mathrm{~cm}^{2} \mathrm{~s}^{-1}$ for NZ-GEC and $1.7010^{-5} \mathrm{~cm}^{2} \mathrm{~s}^{-1}$ for the SZ-GEC sensor. By comparison with the theoretical diffusion coefficient, equal to $6.710^{-6} \mathrm{~cm}^{2} \mathrm{~s}^{-1}$ reported in literature [13], all sensors exhibited higher diffusion coefficients. Electroactive surface area of $1.0210^{-3} \mathrm{~cm}^{2}$ for GEC was the highest from all sensors, value which decreased from GEC to ZN-GEC (5.7 $\left.10^{-4} \mathrm{~cm}^{2}\right)$ and SZ-GEC $\left(3.810^{-4} \mathrm{~cm}^{2}\right)$. In all cases, the electroactive surface areas were higher than the calculated geometric area of $2.510^{-4} \mathrm{~cm}^{2}$. These expected results are due to the use of a decreased concentration of graphite in SZ-GEC and NZ-GEC sensors and increased quantity of insulating materials. Also, these results suggest that the sensors presented a large number of conducting microzones and that the majority of their surface was electroactive [14].

Figure 2 shows comparatively cyclic voltammograms recorded in $2 \mathrm{mM}$ $\mathrm{K}_{3} \mathrm{Fe}(\mathrm{CN})_{6} / \mathrm{K}_{4} \mathrm{Fe}(\mathrm{CN})_{6}$ of all three sensors at the selected optimal composition. The largest peak-to-peak separation together with less developed peaks was obtained for NZ-GEC, with a $\Delta E=0.343 \mathrm{~V}$. Lower peak-to-peak separations, $0.184 \mathrm{~V}$ and $0.093 \mathrm{~V}$, are characteristic for GEC and SZ-GEC, respectively. This favorable behavior close to a reversible system at SZ-GEC presents also well defined peaks. By plotting ferrycianide anodic/cathodic peak current as a function of the scan rate, resulted in a linear relationship of I vs. $\mathrm{v}^{1 / 2}$ in the investigated range (results not shown), indicating a diffusion-controlled process.
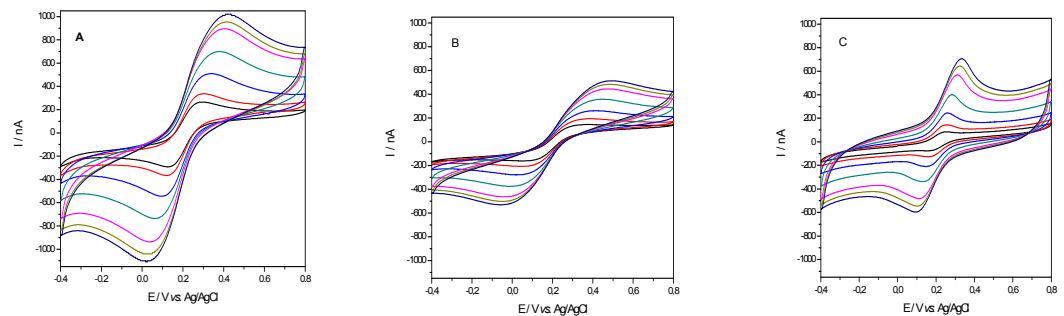

Figure 2: Cyclic voltammograms recorded at (A) GEC, (B) ZN-GEC, (C) SZ- GEC, in the presence of $2 \mathrm{mM}$ potassium ferri/ferrocyanide at different scan rates : 25, 50, 100, 200, 333, $400,454 \mathrm{mV} \mathrm{s}^{-1}$ in $1 \mathrm{M} \mathrm{KNO}_{3}$ supporting electrolyte; potential ranged from -0.4 to $+0.8 \mathrm{~V}$.

Voltammetric performance of the sensors in these experimental conditions can be regarded as starting point for electrochemical behavior characteristics investigation. Literature data state a hypothesis in which sensors fabricated using a mixture of graphite particles and epoxy resin, exhibit a random or ordered 
microelectrode ensemble behavior. From Figure 2, all voltammograms at slow scan rates present a sygmoidal response consistent to a microelectrode behavior. At fast scan rates a more hysteresis behavior is obvious, especially at SZ-GEC sensor, characteristic for solid macroelectrode behavior [15]. Plotting $\log i$ vs. $\log v$, (graphs not shown) for all three composites, slopes equal to 0.475 for GEC, 0.436 for NZ-GEC, and 0.724 for SZ-GEC are obtained, and according to [14], a slope lower than 0.5 is associated to a mixed linear and spherical diffusion, which is characteristic at microelectrodes, depending on experimental time scale.

\subsection{Cyclic voltammetry of ascorbic acid}

Electrochemical behaviour of AA was investigated by $\mathrm{CV}$ at all three carbon composite sensors. For all cyclic voltammograms presented in Figure 3, an anodic peak is observed during the positive scans and no cathodic peak during the negative scans, showing an irreversible oxidation process at all three electrode materials.
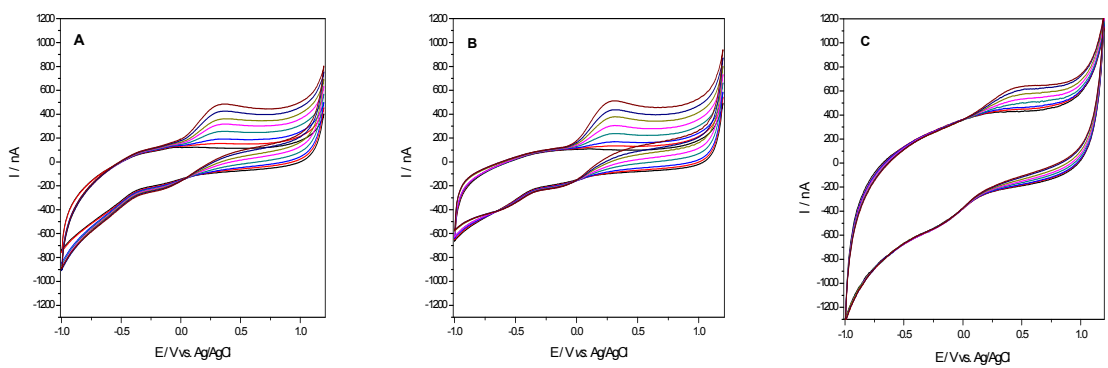

Figure 3: Cyclic voltammograms recorded at (A) GEC, (B) ZN-GEC, (C) SZ-GEC, in $50 \mathrm{mM}$ PBS supporting electrolyte with increasing concentrations of AA : $125 \mu \mathrm{M}, 250 \mu \mathrm{M}, 500 \mu \mathrm{M}, 750 \mu \mathrm{M}, 1 \mathrm{mM}$, $1.25 \mathrm{mM}, 1.5 \mathrm{mM}$; Pt reference electrode and $\mathrm{Ag} / \mathrm{AgCl}$ auxiliary electrode; potential ranged from $-1.0 \mathrm{~V}$ to $+1.2 \mathrm{~V}$; scan rate of $250 \mathrm{mV} \mathrm{s}^{-1}$.

The electrochemical oxidation of AA at GEC (oxidation potential of $0.365 \mathrm{~V}$ ) and ZN-GEC (oxidation potential of $0.303 \mathrm{~V}$ ) presented more sharpen oxidation peaks compared to the broaden peaks encountered at SZ-GEC (oxidation potential at $0.521 \mathrm{~V}$ ). The more negative potential value for AA oxidation at NZGEC in comparison with the bare graphite-epoxy composite sensor (GEC) can be due to the catalytic effect of the zeolite material incorporated in the carbon composite matrix. In the same time, it was observed that the peak current of AA is shifted to a more positive potential value at SZ-GEC compared with the same blank sensing device. Detection potentials of ascorbic acid at similar carbon composite materials have been reported at approximately $0.5 \mathrm{~V} v s$. SCE at a carbon paste electrode [16], at $0.5 \mathrm{~V} v s$. SCE at a carbon ceramic electrode [10], and around $0.65 \mathrm{~V}$ at an unmodified carbon paste electrode [17]. 
The oxidation peak current response was directly proportional to AA concentrations, and linear calibrations with good correlation coefficients were obtained in the range between $125 \mu \mathrm{M}$ and $1.5 \mathrm{mM}$ AA. The sensitivity values of

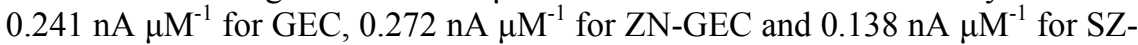
GEC were determined (graphs not shown). The best sensitivity was obtained for the electrode material containing natural zeolite, with a value close to the blank electrode material, while for synthetic zeolite-composite material a lower sensitivity was found.

Taken into consideration the final application of the designed carbon composite materials, the fact that SZ-GEC sensor presented a slightly decreased sensitivity towards AA detection, without any protective membranes at the electroactive sensing surface, makes this material a candidate for further investigations related to the response towards dopamine molecule in the presence of ascorbic acid as main interfering compound.

\subsubsection{Cyclic voltammetry of ascorbic acid and dopamine}

$\mathrm{AA}$ and DA coexist in the extra-cellular fluid of the central nervous system and exhibit similar oxidation potentials at carbon based materials, and the selective determination of these biomolecules is a great problem due to the overlapped signals. A real challenge is given by the in vivo measurement of dopamine in the presence of ascorbic acid, without a significant interference, which requires certain preliminary electrochemical tests.

As preliminary electrochemical tests, in order to notice the dopamine electrochemical response at SZ-GEC, cyclic voltammetry experiments were carried out in the presence of $1.5 \mathrm{mM} \mathrm{AA}$, by adding increasing concentrations of DA until a maximum concentration of $1.5 \mathrm{mM}$ DA was reached. The voltammograms are presented in Figure 4, and more defined peaks are noticed for DA oxidation. Also, a cathodic peak occurred at reversed scanning in the presence of DA, and a difference between anodic and cathodic peak potential equal to $0.360 \mathrm{~V}$, is characteristics to a quasi-reversible redox process.

In comparison with AA oxidation potential value of $0.512 \mathrm{~V}$, the oxidation process of DA occurred at a lower potential value of $0.385 \mathrm{~V}$, which informs that DA oxidation on the electrode surface is favoured against AA electrooxidation. This aspects is confirmed also by a greater sensitivity reached for DA detection

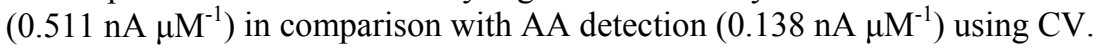

A similar experiment was carried out to explore the electrochemical behaviour of the electrode, first in the presence of same DA concentrations range, and then AA concentrations were added up to $1.5 \mathrm{mM} \mathrm{AA}$. Based on CVs recorded and presented in Fig. 5, the sensitivities for the simultaneous DA and

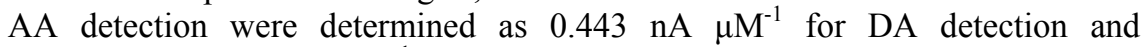
respective, $0.059 \mathrm{nA} \mu \mathrm{M}^{-1}$ for AA detection. For AA detection no good correlation coefficient was obtained, which informed that no AA detection is possible under these working conditions. Also, it must be noticed a small difference between the sensitivities for DA detection under both working 


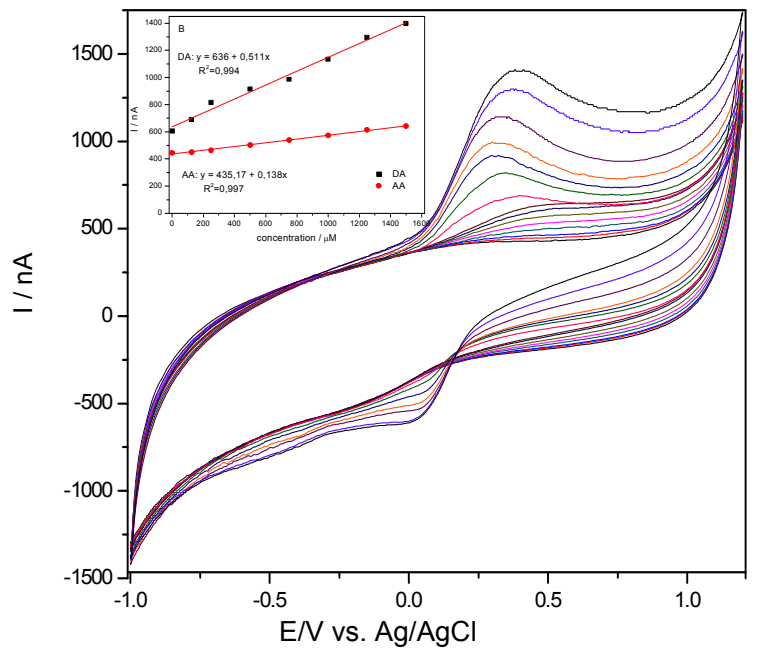

Figure 4: Cyclic voltammograms recorded at SZ-GEC electrode in $50 \mathrm{mM}$ PBS supporting electrolyte with increasing concentrations of AA followed by increasing concentrations of DA: $125 \mu \mathrm{M}, 250 \mu \mathrm{M}$, $500 \mu \mathrm{M}, 750 \mu \mathrm{M}, 1 \mathrm{mM}, 1.25 \mathrm{mM}, 1.5 \mathrm{mM}$ (Inset: calibration plots of the current $v s$. DA concentration at a potential value of $E=+0.385$ $\mathrm{V}$ and AA concentration at a potential value of $E=+0.512 \mathrm{~V}$ ).

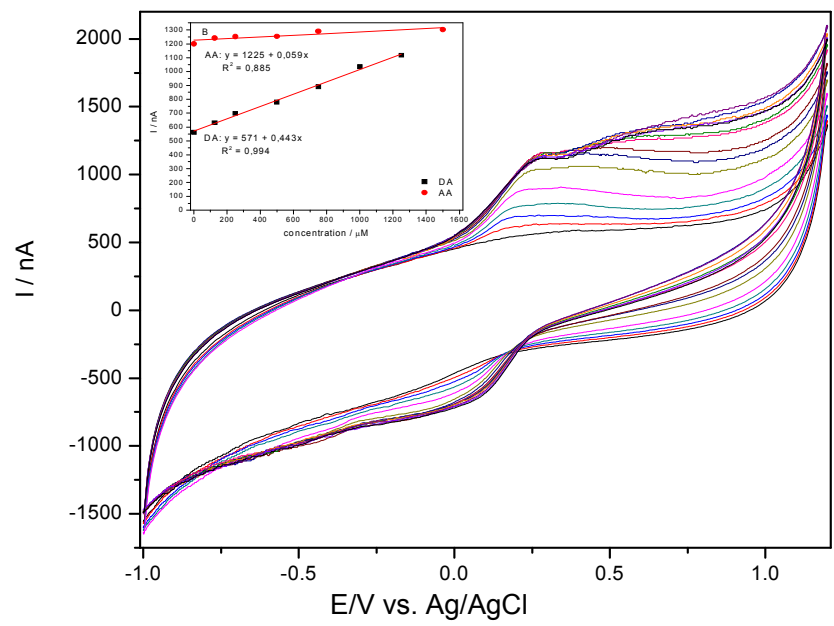

Figure 5: Cyclic voltammograms recorded at SZ-GEC in $50 \mathrm{mM}$ PBS supporting electrolyte with increasing concentrations of DA followed by increasing concentrations of AA: $125 \mu \mathrm{M}, 250 \mu \mathrm{M}$, $500 \mu \mathrm{M}, 750 \mu \mathrm{M}, 1 \mathrm{mM}, 1.25 \mathrm{mM}, 1.5 \mathrm{mM}$ (Inset calibration plots of the current $v s$. DA concentration at a potential value of $E=$ $+0.275 \mathrm{~V}$ and AA concentration at a potential value of $E=+0.521 \mathrm{~V}$. 
conditions. A better DA detection sensitivity was reached under the working

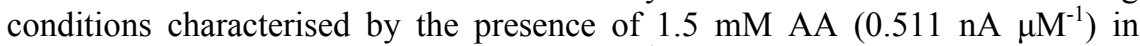
comparison with AA absence $\left(0.443 \mathrm{nA} \mu \mathrm{M}^{-1}\right)$, which means that about $15 \%$ of the sensitivity is owing to AA presence. This aspect should be explained by the fact that in homogeneous solutions, AA can reduce the DA oxidation product back to DA, improving the local concentration of DA at the electrode surface [18]. To avoid AA interference for DA detection in the practical application, either a precalibration of the sensor in AA presence is required or a permselective Nafion membrane should be proposed.

\subsection{Amperometric detection of dopamine}

Constant-potential amperometry (CPA) is a very useful technique for practical applications and offers the best temporal resolution among available techniques. Taking into consideration that in the extracellular fluid of the central nervous system, DA concentrations are present at a level from nanomolar to micromolar range $(0.01-1 \mu \mathrm{M})[19]$, CPA should be regarded as the most viable.

Table 1: $\quad$ Analytical parameters for CPA technique for dopamine detection at microelectrodes.

\begin{tabular}{|c|c|c|c|}
\hline \multirow{2}{*}{ Parameters } & \multicolumn{3}{|c|}{ Electrode type } \\
\cline { 2 - 4 } & GEC & NZ-GEC & SZ-GEC \\
\hline $\begin{array}{c}|c| \\
\text { Potential value[V vs. } \\
\text { Ag/AgCl] }\end{array}$ & +0.250 & +0.150 \\
\hline Sensitivity [nA $\left.\mu M^{-1}\right]$ & 0.0493 & 0.073 & 0.135 \\
\hline Correlation coefficient, $\mathrm{R}^{2}$ & 0.989 & 0.997 & 0.999 \\
\hline LOD $[\mu \mathrm{M}]$ & 101.100 & 39.900 & 0.210 \\
\hline RSD $[\%]$ & 8.900 & 6.500 & 3.550 \\
\hline LQ $[\mu \mathrm{M}]$ & 336.710 & 133.000 & 0.729 \\
\hline
\end{tabular}

Figure 5 presents the amperometric measurements of DA recorded at SZGEC microelectrode at an applied potential of $+0.150 \mathrm{~V}$, while all electroanalytical parameters recorded as well at GEC and NZ-GEC microelectrodes are gathered in Table 1 . The best results were obtained for SZGEC in relation to the electroanalytical parameters, i.e., a limit of detection of

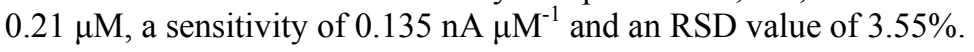

In-vitro detection of low dopamine concentrations must be regarded as starting point for improving the microsensors for further in vivo experiments. Serious interactions may occur at implantation due to the exposure to brain tissue and the study of SZ-GEC microsensor to DA response when covered with specific membranes to assure selectivity has to be taken into consideration for future studies. 


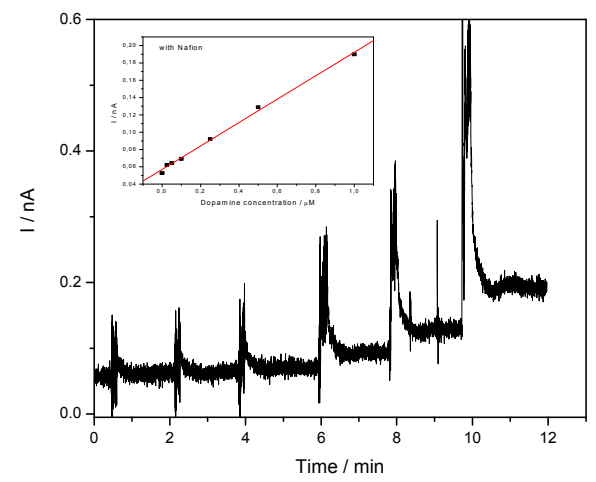

Figure 6: $\quad$ CPA recoded at SZ-GEC microelectrode in $50 \mathrm{mM}$ PBS supporting electrolyte with increasing concentrations of Dopamine : $25 \mathrm{nM}$, $50 \mathrm{nM}, 100 \mathrm{nM}, 250 \mathrm{nM}, 500 \mathrm{nM}, 750 \mathrm{nM}, 1 \mu \mathrm{M}$; Pt reference electrode and $\mathrm{Ag} / \mathrm{AgCl}$ auxiliary electrode; Potential value in $+0.150 \mathrm{~V}$; inset is presented the calibration plot of DA oxidation at potential value of $+0.150 \mathrm{mV}$.

\section{Conclusions}

Graphite-epoxy (GEC), natural zeolite-graphite-epoxy (NZ-GEC), synthetic zeolite-graphite-epoxy (SZ-GEC) composite microelectrodes were obtained and tested for ascorbic acid (AA) and dopamine (DA) detection. The compositions of the microelectrodes were optimized as $55 \%$ graphite and $45 \%$ wt. epoxy for GEC and graphite 45\%-natural/synthetic zeolite 20\%-epoxy 35\%, wt. for both NZ-GEC and SZ-GEC in according with the electrochemical properties determined by classical ferro/ferricyanide system. ZN-GEC and GEC microelectrodes exhibited very good sensitivity for AA detection in aqueous solution. The slowest kinetics of AA oxidation and the fast kinetics of DA oxidation on SZ-GEC recommend it as appropriate for practical potential applications for DA detection in the presence of AA.

\section{Acknowledgements}

This work was partially supported by the strategic grants, POSDRU 2009 project ID 50783, POSDRU/89/1.5/S/63700, POSDRU 107/1.5/S/77265, POSDRU project ID 57649(PERFORM-ERA) co-financed by the European Social FoundInvesting in People, and partially by the PN-II- ID-PCE-165/2011. 


\section{References}

[1] Stulik, K., Amatore, C., Holub, K., Marecek, V. and Kutner, W. Microelectrodes. Definitions, characterization, and applications. Pure and applied Chemistry, 72, pp. 1483-1492, 2000.

[2] Bolger, F.B, McHugh, S.B., Bennett, R., Li, J., Ishiwari, K. Francois, J., Conway, M.W., Gilmour, G., Bannerman, D.M., Fillenz, M., Tricklebank, M. and Lowry, J.P., Characterisation of carbon paste electrodes for realtime amperometric monitoring of brain tissue oxygen. Journal of Neuroscience methods, 195, pp. 135-142, 2011.

[3] Huang, X., Pot, J.J. and Kok, W.T., Electrochemical characteristics of conductive carbon cement as matrix for chemically modified electrodes. Analytica Chimica Acta, 300, pp. 5-14, 1995.

[4] Pumera, M., Merkoci, A. and Alegret, S., Carbon nanotube-epoxy composites for electrochemical sensing. Sensors and Actuators B, 113, pp. 617-622, 2006.

[5] Kalcher, K., Svancara, I., Buzuk, M., Vytras, K. and Walcarius, A., Electrochemical sensors and biosensors based on heterogeneous carbon materials. Springer-Verlag, pp. 861-889, 2009.

[6] Chang, M. and Chang, C., Voltammetric determination of sunscreen by convenient epoxy-carbon composite electrodes. Journal of Food and Drug Analysis, 9(4), pp. 199-206, 2001.

[7] Carvalho, R.H., Lemos, F., Lemos, M.A.N.D.A., Cabral, J.M.S. and Riberio, F.R., Electro-oxidation of phenol on zeolite/graphite composite electrodes Part1. Electrochemical behaviour over NaY zeolite. Journal of Molecular Catalysis A: Chemical, 248, pp. 48-52, 2006.

[8] Marakani, M., Akrami, Z., Kazemian, H. and Zare, H.R., Electrocatalytic characteristics of uric acid oxidation at graphite-zeolite-modified electrode doped with iron (III). Journal of Electroanalytical Chemistry, 586, pp. 3138, 2006.

[9] Auerbach, S.M., Carrado, K.A. and Dutta, P.K., Handbook of zeolite science and technology. Mercel Dekker: New York, pp. 754-918, 2003.

[10] Chang, M. and Chang, C., Simultaneous voltammetric determination of ascorbic acid and its derivates in cosmetics using epoxy-carbon composite electrodes. Journal of Food and Drug Analysis, 13(3), pp. 205-211, 2005.

[11] Nezamzadeh, A., Amini, M.K. and Faghihian, H., Square-wave voltammetric determination of ascorbic acid based on its electrocatalytic oxidation at zeolite-modified carbon paste electrodes. International Journal of Electrochemical Science, 2, pp. 583-594, 2007.

[12] Orha, C., Pop, A., Lazau, C., Grozescu, I., Tiponut, V. and Manea, F., Structural characterization and the sorption properties of the natural and synthetic zeolite. Journal of optoelectronics and advanced materials, 13(5), pp. 544-549, 2011.

[13] Kenopka, S.J. and McDuffle, B., Diffusion coefficient of feri-and ferrocyanide ions in aqueous media, using twin electrode thin layer electrochemistry. Analytical Chemistry, 42, pp. 1741-1746, 1970. 
[14] Garcia, S.R., Alagret, S., Cespedes, F. and Forster, R.J., Carbon composite electrodes: surface and electrochemical properties. Analyst, 127, pp. 1512$1519,2002$.

[15] O'Hare, D., Macpherson, J.V. and Willows, A., On the microelectrode behaviour of graphite-epoxy composite electrodes. Electrochemistry Communications, 4, pp. 245-250, 2002.

[16] Salimi, A., MamKhezri, H. and Hallaj, R., Simultaneous determination of ascorbic acid, uric acid and neurotransmitters with a carbon ceramic electrode prepared by sol-gel technique. Talanta, 70, pp. 823-832, 2006.

[17] Raoof, J.B., Ojani, R. and Kiani, A., Carbon paste electrode spiked with ferrocene carboxylic acid and its application to the electrocatalytic determination of ascorbic acid. Journal of Electroanalytical Chemistry, 515, pp. 45-51, 2001.

[18] Rocha, L.S. and Carapuca, H.M., Ion-exchange voltammetry of dopamine at Nafion-coated glassy carbon electrodes: Quantitative features of ionexchange partition and reassessment on the oxidation mechanism of dopamine in the presence of excess ascorbic acid. Bioelectrochemistry, 69, pp. 258-266, 2006.

[19] Capella, P., Ghasemzadeh, B., Mitchell, K. and Adams, R.N., Nafioncoated carbon fiber electrodes for neurochemical studies in brain tissue. Electroanalysis, 2, pp. 175-182, 1990. 